\title{
An Integer Grey Goal Programming For Project Time, Cost and Quality Trade-Off
}

\author{
Seyed Hossein Razavi Hajiagha ${ }^{1}$, Hadi Akrami², Shide Sadat Hashemi ${ }^{3}$, Hannan Amoozad \\ Mahdiraji $^{3}$
}

${ }^{1}$ Khatam Institute of Higher Education

No. 30, Hakim Azam Ave., North Shiraz Ave., Mollasadra Street, Tehran, IRAN

E-mail.s.hossein.r@gmail.com

${ }^{2}$ EN Bank

No. 24,Esfandiyar Blvd., Valiasr Ave., 1968655944, Tehran, IRAN

E-mail.h.akrami@gmail.com

${ }^{3}$ Department of Management

Kashan Branch, Islamic Azad University, Kashan, Iran

E-mail.shide_hashemi@yahoo.com,h.amoozad@ut.ac.ir

cross $^{\text {ref }}$ http://dx.doi.org/10.5755/j01.ee.26.1.4633

\begin{abstract}
Project management (PM) is one of the prominent fields in business and industry. Every task of an organization can be imagined as a project, being a coordinated set of activities toward a common goal. One important aspect of PM is analysing the information related to the optimum balance among the project's objectives. Each project is a combination of different activities, being connected to each other and having several success criteria, among which the time, cost and quality of the project completion are more significant, due to their significant effect on obtained results. Accordingly, the time might lead to delay and penalty which means more cost; and cost may be underestimated than real required funds. They both will lead to failure in project management. On the other hand, quality is the final key which confirms the success. The aim of a time-cost-quality trade-off problem (TCQTP) is to select a set of activities and an appropriate execution mode for each activity; the cost and time of the project is minimized while the project quality is maximized. The purpose of this paper is to present a model for TCQTP in which these parameters are approximated by grey numbers. Since there are various modes to accomplish each activity, the trade-off problem is formulated based upon a multiobjective integer grey programming model. Afterwards, a goal programming-based approach is designed to solve this model. The model's results provide a framework for the project manager to manage his/ her project successfully, in acceptable time, with the lowest cost and the highest quality. The main originality of the proposed model is the approximation of time, cost and quality parameters of activities mode with grey numbers and the development of a two phase goal programming- based approach to solve this problem. Ultimately, the proposed model is applied in two different cases and results are illustrated to clarify the outstanding capabilities of the model.
\end{abstract}

Keywords: the iron triangle, project management, time, cost and quality trade-off; grey numbers; integer goal programming.

\section{Introduction}

Project management is one of the most important fields in business and industry. Every task in an organization can be taken into account as a project, i.e. a temporary endeavour undertaken to produce a unique product, service or result (Lewis, 2010). In this context, the purpose of the project management is to foresee or predict as many dangers and problems as possible and to plan, organize, and control activities so that projects are completed successfully in spite of all the risks (Lock, 2007). Many have attempted to define project management. According to PMBOK Guide, the nine knowledge areas in project management are project integration, time, cost, quality, human resources, communication, risk and procurement management (PMI, 2008). Research studies investigating the reasons why projects fail, provide lists of factors believed to contribute to the project management success or failure. There are three main points that are the most important to a successful project: (1) a project must meet the customer requirements, (2) it has to be within budget, and (3) it has to be on time (Rasmy, 2008). These three criteria are often referred to as The Iron Triangle (Atkinson, 1999).

One important aspect of project management is to know about the information related to the optimum balance between the project's objectives. According to the iron triangle, time, cost and quality are important objectives of a project. Heretofore, extensive research to develop costtime trade-off problems has been conducted. Nowadays the quality of a project is also added to the project time and cost. The aim of a time-cost-quality trade-off problem (TCQTP) is to select a set of activities and an appropriate execution mode for each activity so that the cost and time of the project is minimized while the project quality is maximized (Shahsavari-Pour et al., 2010). 
The problem of the project's time-cost trade-off was first studied by (Kelly, 1961). He assumed a linear relation between time and cost of an activity and offered a mathematical modelling and a heuristic algorithm (but one that leads to optimal solutions) to solve the problem. By assuming that the direct cost of an activity varies with time, mathematical programming models were developed to minimize the project's direct cost (Babu \& Suresh, 1996). Many researchers have developed mathematical programming model for the cost and time trade-off problems hereafter.

(Khosrowshahi, 1995) presented the relationship between project's total cost and project's total duration for a given type of project and represented this relationship in mathematical form. (Phillips, 1996) presented an application oriented procedure to solve the project management duration/ resource trade-off problem. A procedure is presented to reduce a project from a normal to a crash duration state at a minimum amount of additional resource expenditure assuming a linear utilization function. The procedure was a network based on using a graphical cut search approach to locate the minimal resource level at each reduction in total project duration. (Babu \& Suresh, 1996) were the first who suggested that the quality of a completed project may be affected by project crashing. For the sake of simplicity, they adapted the continuous scale from zero to one to specify quality attained at each activity. The overall project quality is a function of quality levels attained at the individual activities. They developed optimization models involving the project time-costquality trade-off which would assist in expediting a project weighing time-cost-quality triangle. Each of the three developed models optimizes one of these three entities by assigning desired levels (bounds) on the two others. (Demeulemeester et al., 1998) described a new exact procedure for the discrete time/cost trade-off problem in deterministic activity-on-the-arc networks of the CPM type, where the duration of each activity is a discrete, nonincreasing function of the amount of a single resource (money) committed to it. The objective is to construct a complete and efficient time/cost profile over the set of feasible project durations. (AbdelSalam \& Bao, 2000) implemented a linear programming formulation to solve this problem through a truly interactive computation environment. (Vanhoucke, 2007) presented an electromagnetic meta-heuristic algorithm for the discrete time/cost trade-off problem. (Cohen et al., 2007) considered the problem of allocating resources to projects performed under given due dates and stochastic time-cost trade-off settings. They showed how to implement a stateof-the-art methodology known as "robust optimization" to solve the problem in particular. (Abbasnia et al., 2008) investigated a new approach in solving time-cost trade-off problem, because of uncertainties which affect activity cost. Fuzzy logic theory is employed to consider uncertainties affecting total direct and indirect cost of a construction project. Non-dominated Sorting Genetic Algorithm (NSGA) is applied to provide a trade-off between time and total cost. (Iranmanesh et al., 2008) tried to determine optimal solutions from which the project manager will select his desirable choice to run the project. Their problem was multi-objective and the purpose was to find the Pareto optimal front of time, cost and quality of a project and a meta-heuristic method is developed based on a version of genetic algorithm specially adapted to solve multi-objective problems namely fast PGA. (Mokhtari \& Aghaie, 2009) determined the optimal levels of activity durations and activity costs, which satisfied the project goal(s), led to a balance between the project completion time and the project total cost. In this paper, TCTP (Timecost trade off problem) would be studied considering the influence of discount on the re-source price, using genetic algorithm (GA). (Senouci \& El-Rayes, 2009) presented a multi objective optimization model that provides new and unique capabilities including generating and evaluating optimal/near-optimal construction resource utilization and scheduling plans that simultaneously minimize the time and maximize the profit of construction projects. (Blaszczyk \& Nowak, 2009) analyzed a project-scheduling problem including time-cost and trade-offs proposing a new technique based on computer simulation and interactive approach. (Nikoomaram et al., 2010) developed a model by attending to time value of money (TVOM), which was not considered in previous research. Shahsavari (Pour et al., 2010) developed a model for discrete time-cost-quality trade-off problem that uses the planner-specified weights for handling a multi-objective optimization problem. They proposed a new metaheuristic-based genetic algorithm, called NHGA, for optimizing a multi-objectives time-costquality trade-off problem and analyzed it by the analysis of variance (ANOVA) method. (Razavi Hajiagha et al., 2014) developed a multi objective model of time, cost and quality trade-off in continuous mode.

The main contribution of this work is to consider the uncertainty concept in TCQTP. As (Riabecke, 2006) suggests, under many conditions, exact data are inadequate to model the real-life situations. These situations are called as uncertainty and many researchers developed some structures such as bounded data, ordinal data, fuzzy data, and grey numbers in response to such situations. In fact, most of the decisions aren't made on the basis of wellknown calculations and there is a lot of ambiguity and uncertainty in decision making problems. This condition can occur for a trade-off problem in which the analyst has to estimate the cost, time and quality approximations of a project's activities. Estimation of these parameters by exact and crisp values is difficult and perhaps impossible. Furthermore, this can limit the applications of TCQTP models and reduces its accuracy and coincidence with reality. Therefore, a new model for considering the impreciseness of environmental coefficients is suggested in this study. This study proposes a method which uses a fuzzy grey goal programming (FGGP) approach to model the TCQTP. In this FGGP model, goals values are defined as fuzzy numbers, while parameters (including objectives and constraints coefficients) are specified by grey numbers.

The rest of the paper is organized as followings: section 2 briefly introduces grey numbers. Section 3 presents the process of problem modelling. The problem solving approach is explained in section 4 . Then two illustrative examples are solved in section 5. Finally Section 6 consists of conclusions and suggestions for future work. 


\section{Grey Systems Theory}

Deng (1982) developed the Grey systems theory and presented grey decision making systems (Deng, 1989). Many researchers applied this concept in decision making problems. In simple words, a grey number is a number whose exact value is unknown, but a range within which the value lies is known (Lin et al., 2004). Such a number instead of its range whose exact value is unknown is referred to as a grey number. In applications, a grey number in fact stands for an indeterminate number that takes its possible value within an interval or a general set of numbers. This grey number is generally written as " $\otimes "$. There are several types of grey numbers, but we have only defined interval grey numbers here. This kind of grey number $\otimes_{\text {is }}$ written: $\otimes(a) \in\lfloor\underline{a}, \bar{a}\rfloor$, where $\underline{a}$ stands for the definite, the known lower bound and $\bar{a}$ stands for the definite, the known upper bound of $\otimes(a)$ and $\otimes(a)$ takes its number in this interval (Liu \& Lin, 2010). A comprehensive review on Arithmetic operations of grey numbers is illustrated by (Liu \& Lin, 2006). The main algebraic operations are defined on grey numbers as follows.

Definition1. Let $\otimes(a) \in[\underline{a}, \bar{a}\rfloor$ and $\otimes(b) \in[\underline{b}, \bar{b}]$ be two grey numbers. Then,

$$
\begin{array}{r}
\otimes G_{1}+\otimes G_{2}=\left\lfloor\underline{G}_{1}+\underline{G}_{2}, \bar{G}_{1}+\bar{G}_{2}\right\rfloor \\
\otimes G_{1}-\otimes G_{2}=\left\lfloor\underline{G}_{1}-\underline{G}_{2}, \bar{G}_{1}-\bar{G}_{2}\right\rfloor \\
\otimes G_{1} \times \otimes G_{2}=\left[\begin{array}{l}
\min \left(\underline{G}_{1} \underline{G}_{2}, \underline{G}_{1} \bar{G}_{2}, \bar{G}_{1} \underline{G}_{2}, \bar{G}_{1} \bar{G}_{2}\right), \\
\left.\max \left(\underline{G}_{1} \underline{G}_{2}, \underline{G}_{1} \bar{G}_{2}, \bar{G}_{1} \underline{G}_{2}, \bar{G}_{1} \bar{G}_{2}\right)\right]
\end{array}\right. \\
\otimes G_{1} \div \otimes G_{2}=\left[\underline{G}_{1}, \bar{G}_{1}\right] \times\left[\frac{1}{\bar{G}_{2}}, \frac{1}{\underline{G}_{2}}\right]
\end{array}
$$

Definition2. For grey number $\otimes(x) \in[a, b]$, that:

$$
\begin{aligned}
& \otimes(x) \geq 0 \text { iff } a \geq 0 \text { and } b \geq 0 \\
& \otimes(x) \leq 0 \text { iff } a \leq 0 \text { and } b \leq 0
\end{aligned}
$$

Definition3. For a grey number $\otimes(x) \in[a, b]$, the centre and width can be defined as follows:

$$
\begin{aligned}
& \text { Center : } x_{c}=\frac{1}{2}(a+b) \\
& \text { Width : } x_{w}=\frac{1}{2}(b-a)
\end{aligned}
$$

\section{Model Construction}

A project is represented by a directed acyclic graph $G=(V, E)$ consisting of $m$ nodes and $n$ arcs, in which $V=\{1,2, \ldots, m\}$ is the set of nodes and $E=\{(i, j), \ldots,(l, m)\}$ is the set of direct arcs. Arcs and nodes represent activities and events, respectively. Each project activity, say $(i, j) \in E$, can be executed by a set of modes, $M_{i j}$. Each $k \in M_{i j}$ needs a grey execution time of $\otimes t_{i j k}$, grey cost of $\otimes c_{i j k}$ and grey quality of $\otimes q_{i j k}$. For different values of $k$, $\otimes t_{i j k}, \otimes c_{i j k}$ and $\otimes q_{i j k}$ take different values.
The aim of this paper is to obtain the optimal combination $\left(\otimes t_{i j k}, \otimes c_{i j k}\right.$ and $\left.\otimes q_{i j k}\right)$ of each activity to crash the project network. And while the cost and time of the project is minimized, the project quality is maximized. Notations used for problem formulation are as follow:

$n$ : Number of activities

$M_{i j}$ : Set of available execution modes for activity $i j$, where $i j \in E$;

$\otimes C_{i j k}$ : Direct cost of activity $i j$ if performed by execution mode $k$;

$\otimes t_{i j k}$ : Duration of activity $i j$ if performed by execution mode $k$;

$\otimes q_{i j k}$ : Quality of activity $i j$ if performed by execution mode $k$;

$\otimes Q_{i j}$ : Quality of activity $i j$ in normal duration;

$\otimes q_{i j}$ : Quality of activity $i j$ in crashed duration.

The proposed model's variables are:

$\otimes s_{i}$ : start time of activity $i j$.

$y_{i j k}:\left\{\begin{array}{l}1 \text { :if activity } i j \text { is done in mode } k \\ 0 \text { :otherwise }\end{array}\right.$

The mathematical model of problem is presented as:

$$
\begin{aligned}
& \operatorname{MinC}=\sum_{i j \in E} \sum_{k \in M_{i j}} \otimes C_{i j k} Y_{i j k} \\
& \operatorname{MinT}=\otimes s_{n}-\otimes s_{1} \\
& \operatorname{Max} Q=\frac{\sum_{i j \in E} \sum_{k \in M_{i j}} y_{i j k} q_{i j k}}{n} \\
& S . T . \\
& \otimes s_{j}-\otimes s_{i} \geq \sum_{k \in M_{i j}} \otimes t_{i j k} y_{i j k}, \forall\{i j\} \in E \\
& \sum_{k \in M_{i j}} y_{i j k}=1, \forall\{i j\} \in E \\
& y_{i j k}=0,1
\end{aligned}
$$

The model (7) is a grey multi objective mixed integer (GMOMI) model. To solve this model, a transformation approach is used.

\section{Solving Approach}

In CTQTP there are a number of execution modes to select for each activity. If the number of project activity is $\mathrm{n}$ and there are $\mathrm{k}$ modes for each activity to choose from, then there is $k^{n}$ solution, which results in a very large search space. The complexity of the examined problem is increased when the activities parameters are considered as grey numbers. Therefore, it is necessary to develop some efficient methods to solve the problem. Some papers are devoted to design procedures to solve multi objective integer programming problems (Liu et al., 2000; Jahanshaloo et al., 2003, 2005). Here, a method is developed to solve the model (7) based on approach (Charnes \& Cooper, 1961). The goal programming approach tries to find an optimal solution which has the least deviation from a set of target points. Therefore, a main step in this approach is to determine a set of targets for individual objectives. The proposed approach for 
TCQT problem is therefore composed of two steps: (1) determination of target points for individual objectives, (2) development and solving the goal programming model to find the compromise solution of the entire problem.

\section{Determination of Target Points}

In the first step, three distinct models are solved, for each one of objectives. In fact, there are three grey integer programming models. To obtain an optimal range for each individual grey problem, each problem is solved under two boundary conditions, one for the best condition and one for the worst condition. In the best condition, each activity is done in its best form with the lower bound time, lower bound cost, and upper bound quality. This problem is called optimistic model. In this form, the problem (7) is transformed as follows:

$$
\begin{aligned}
& \operatorname{Min} \underline{C}=\sum_{i j \in E} \sum_{k \in M_{i j}} c_{i j k} Y_{i j k} \\
& \operatorname{Min} \underline{T}=\underline{s}_{n}-\underline{s}_{1} \\
& \operatorname{Max} \bar{Q}=\frac{\sum_{i j \in E} \sum_{k \in M_{i j}} y_{i j k} \bar{q}_{i j k}}{n}
\end{aligned}
$$$$
\text { S.T. }
$$

$$
F S:\left\{\begin{array}{l}
\underline{s}_{j}-\underline{s}_{i} \geq \sum_{k \in M_{i j}}{ }_{t i j k} y_{i j k}, \forall\{i j\} \in E \\
\sum_{k \in M_{i j}} y_{i j k}=1, \forall\{i j\} \in E \\
y_{i j k}=0,1
\end{array}\right.
$$

Now, by solving each objective with the set of FS constraints, the $\underline{C}^{*}, \underline{T}^{*}$, and $\bar{Q}^{*}$ are determined. In the next step, activities are considered in the worst possible condition with the upper bound time and cost and the lower bound quality. A model similar to model (8) is constructed as follows:

$$
\begin{aligned}
& \operatorname{Min} \bar{C}=\sum_{i j \in E} \sum_{k \in M_{i j}} \bar{c}_{i j k} Y_{i j k} \\
& \operatorname{Min} \bar{T}=\bar{s}_{n}-\bar{s}_{1} \\
& \operatorname{Max} \underline{Q}=\frac{\sum_{i j \in E} \sum_{k \in M_{i j}} y_{i j k} \underline{q}_{i j k}}{n} \\
& \text { S.T. } \\
& F S^{\prime}:\left\{\begin{array}{l}
\bar{s}_{j}-\bar{s}_{i} \geq \sum_{k \in M_{i j}} \bar{t}_{i j k} y_{i j k}, \forall\{i j\} \in E \\
\sum_{k \in M_{i j}} y_{i j k}=1, \forall\{i j\} \in E \\
y_{i j k}=0,1
\end{array}\right.
\end{aligned}
$$

Solving individual objectives of model (9) with the $F S^{\prime}$ constraints, the $\bar{C}^{*}, \bar{T}^{*}$, and $\underline{Q}^{*}$ are determined.

\section{Goal Programming Formulation}

Consider the problem (7). The target values for cost, time and quality objectives are determined as $\left(\underline{C}^{*}, \bar{C}^{*}\right)$, $\left(\underline{T}^{*}, \bar{T}^{*}\right)$, and $\left(Q^{*}, \bar{Q}^{*}\right)$, respectfully. In this step, a goal programming model is developed to minimize the undesired deviations from target values as follows:

$$
\begin{aligned}
& \operatorname{Mind}_{1}^{+}+d_{2}^{+}+d_{3}^{+}+d_{4}^{+}+d_{5}^{-}+d_{6}^{-} \\
& \sum_{i j \in E} \sum_{k \in M_{i j}} \underline{c}_{i j k} y_{i j k}-d_{1}^{+}+d_{1}^{-}=\underline{C}^{*} \\
& \sum_{i j \in E} \sum_{k \in M_{i j}} \bar{c}_{i j k} y_{i j k}-d_{2}^{+}+d_{2}^{-}=\bar{C}^{*} \\
& \underline{\mathrm{s}}_{\mathrm{n}}-\underline{s}_{1}-d_{3}^{+}+d_{3}^{-}=\underline{T}^{*} \\
& \overline{\mathrm{s}}_{\mathrm{n}}-\overline{\mathrm{s}}_{1}-d_{4}^{+}+d_{4}^{-}=\bar{T}^{*} \\
& \sum_{i j \in E} \sum_{k \in M_{i j}} \underline{q}_{i j k} y_{i j k}-d_{5}^{+}+d_{5}^{-}=\underline{Q}^{*} \\
& \sum_{i j \in E} \sum_{k \in M_{i j}} \bar{q}_{i j k} y_{i j k}-d_{6}^{+}+d_{6}^{-}=\bar{Q}^{*}
\end{aligned}
$$

S.T.

$\mathrm{Y} \in \mathrm{FS}$

$\mathrm{Y} \in \mathrm{FS}^{\prime}$

$\mathrm{d}_{\mathrm{i}}^{+}, \mathrm{d}_{\mathrm{i}}^{-} \geq 0, i=1,2, \ldots, 6$

Where, the $d_{i}^{-}$and $d_{i}^{+}, i=1,2, \ldots, 6$ are the negative and positive deviations from target values. The objective function (15) tries to minimize the total deviation from target values. The project manager may have some preferences over different objectives. In this case, he/ she can introduce some weights in objective function. Therefore, the objective function is transformed as follows:

$$
\operatorname{Min} \sum_{k=1}^{3} p_{k}\left(\sum_{i \in P_{k}} d_{i}^{+} \vee d_{i}^{-}\right)
$$

Where, $\quad p_{k} \succ \succ p_{k+1}, \quad P_{k}$ is the objectives in $k^{\text {th }}$ preference, and " $\vee$ " is the "logical or" operator. The model (15) is an integer linear programming problem which can be easily solved with available applications. The optimal solution of this model determined the best feasible solution with the lowest deviation from optimal individual solutions. The computational complexity of the algorithm is associated with solving a mixed integer or pure $0 / 1$ problem which can be solved with ordinal optimization packages in a reasonable time.

A critical issue in multi-objective problems is to find a Pareto optimal or efficient set of solutions. In a multiobjective problem with $k$ objectives $f_{l}(x), l=1,2, \ldots, k$ over a feasible space $X$, a solution $x_{0} \in X$ is called Pareto optimal if and only if there does not exist another $y \in X$ such that $f_{l}(y) \geq f_{l}\left(x_{0}\right)$ for all $l$ and $f_{l}(y)>f_{l}\left(x_{0}\right)$ for at least one $p$ (Abraham et al., 2006).in fact, a solution $x_{0}$ is Pareto optimal if and only if there does not exist another solution $y$ that performed equally in all objectives and outperformed it at least in one objective.

It now remains to show that the solution obtained from solving model (10) is a Pareto optimal solution of multiobjective TCQTP. This is done in the following lemma.

Lemma. If $y_{0}^{*}$ is an optimal solution of model (10), it will be a Pareto optimal solution of multi-objective TCQTP in model (7). 
Proof. On the contrary, suppose that $y_{0}^{*}$ is not a Pareto optimal solution of model (7). Therefore, there is another solution $y_{1}$ that outperformed $y_{0}^{*}$. Without loss of generality, suppose that $y_{1}$ and $y_{0}^{*}$ has equal time and quality, i.e. $T_{y_{1}}=T_{y_{0}^{*}}$ and $Q_{y_{1}}=Q_{y_{0}^{*}}$, while it has a lower cost, i.e. $C_{y_{1}}<C_{y_{0}^{*}}$. Therefore, $d_{3 y_{1}}^{+}=d_{3 y_{0}^{*}}^{+}, d_{4 y_{1}}^{+}=d_{4 y_{0}^{*}}^{+}$, $d_{5 y_{1}}^{-}=d_{5 y_{0}^{*}}^{-}$and $d_{6 y_{1}}^{-}=d_{6 y_{0}^{*}}^{-}$. However, since $C_{y_{1}}<C_{y_{0}^{*}}$, then it must hold that $d_{1 y_{1}}^{+}<d_{1 y_{0}^{*}}^{+}$or $d_{2 y_{1}}^{+}<d_{2 y_{0}^{*}}^{+}$. It follows that:

$$
\begin{aligned}
& \left(d_{1 y_{1}}^{+}+d_{2 y_{1}}^{+}+d_{3 y_{1}}^{+}+d_{4 y_{1}}^{+}+d_{5 y_{1}}^{-}+d_{6 y_{1}}^{-}\right)< \\
& \left(d_{1 y_{0}^{*}}^{+}+d_{2 y_{0}^{*}}^{+}+d_{3 y_{0}^{*}}^{+}+d_{4 y_{0}^{*}}^{+}+d_{5 y_{0}^{*}}^{-}+d_{6 y_{0}^{*}}^{-}\right)
\end{aligned}
$$

That contradicts with the optimality of $y_{0}^{*}$ for model (10). It completes the proof.

\section{Illustrative Examples}

Example1. First consider an illustrative example, include 3 activities, which is shown in figure 1 .

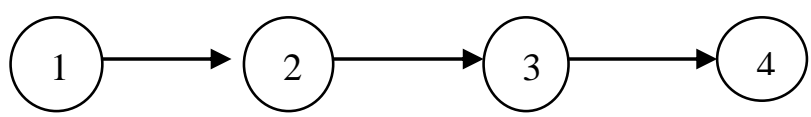

Figure1. An illustrative network consisting of three activities Each activity has two different modes which are presented in table 1. According to table 1, the TCQT model, Eq. (7) can be written as Eq. (12).

Table 1

Activities execution parameters in different modes

\begin{tabular}{|c|c|c|}
\hline \multirow{2}{*}{ Activity } & \multicolumn{2}{|c|}{ Modes } \\
\cline { 2 - 3 } & 1 & 2 \\
\hline $1-2$ & $([2,4][3,5][0.7,0.8])$ & $([1,2][2,4][0.8,0.9])$ \\
\hline $2-3$ & $([2,3][4,5][0.8,0.9])$ & $([5,6][6,7][0.8,0.9])$ \\
\hline $3-4$ & $([3,5][6,7][0.7,0.8])$ & $([5,7][8,9][0.7,0.8])$ \\
\hline
\end{tabular}

$\operatorname{Min} C=[3,5] y_{121}+[2,4] y_{122}+[4,5] y_{231}+[6,7] y_{232}$

$+[6,7] y_{341}+[8,9] y_{342}$

$\operatorname{MinT}=\left[\underline{s}_{4}, \bar{s}_{4}\right]-\left[\underline{s}_{1}, \bar{s}_{1}\right]$

$\operatorname{Max} Q=\left([0.7,0.8] y_{121}+[0.8,0.9] y_{122}+[0.8,0.9] y_{231}\right.$

$\left.\left.+[0.8,0.9] y_{232}+[0.7,0.8] y_{341}+[0.7,0.8] y_{342}\right) / 3\right)$

S.T.

$\left[\underline{s}_{2}, \bar{s}_{2}\right]-\left[\underline{s}_{1}, \bar{s}_{1}\right] \geq[2,4] y_{121}+[1,2] y_{122}$

$\left[\underline{s}_{3}, \bar{s}_{3}\right]-\left[\underline{s}_{2}, \bar{s}_{2}\right] \geq[2,3] y_{231}+[5,6] y_{232}$

$\left[s_{4}, \bar{s}_{4}\right]-\left[s_{3}, \bar{s}_{3}\right] \geq[3,5] y_{341}+[5,7] y_{342}$

$y_{121}+y_{122}=1$

$y_{231}+y_{232}=1$

$y_{341}+y_{342}=1$

$y_{121}+y_{122}, y_{231}, y_{232}, y_{341}, y_{342} \in\{0,1\}$
In this example, total number of execution modes is $2^{3}=8$ and the optimal solution can easily be obtained by complete enumeration. The Eq. (8) for this example is constructed as follows:

$$
\begin{aligned}
& \operatorname{Min} \underline{C}=3 y_{121}+2 y_{122}+4 y_{231}+6 y_{232} \\
& +6 y_{341}+8 y_{342} \\
& \operatorname{Min} \underline{T}=\underline{s}_{4}-\underline{s}_{1} \\
& \operatorname{Max} \bar{Q}=\left(0.8 y_{121}+0.9 y_{122}+0.9 y_{231}\right. \\
& \left.+0.9 y_{232}+0.8 y_{341}+0.8 y_{342}\right) / 3
\end{aligned}
$$

S.T.

$$
F S:\left\{\begin{array}{l}
\underline{s}_{2}-\underline{s}_{1} \geq 2 y_{121}+y_{122} \\
\underline{s}_{3}-\underline{s}_{2} \geq 2 y_{231}+5 y_{232} \\
\underline{s}_{4}-\underline{s}_{3} \geq 3 y_{341}+5 y_{342} \\
y_{121}+y_{122}=1 \\
y_{231}+y_{232}=1 \\
y_{341}+y_{342}=1 \\
y_{121}+y_{122}, y_{231}, y_{232}, y_{341}, y_{342} \in\{0,1\}
\end{array}\right.
$$

By solving three distinct problems to minimize the $\underline{C}$, $\underline{T}$, and $\bar{Q}$, the solutions will be $\underline{C}^{*}=12, \underline{T}^{*}=6$, and $\bar{Q}^{*}=0.867$. Similarly, the pessimistic model is formulated as Eq. (14):

$$
\begin{aligned}
& \operatorname{Min} \bar{C}=5 y_{121}+4 y_{122}+5 y_{231} \\
& +7 y_{232}+7 y_{341}+9 y_{342} \\
& \operatorname{Min} \underline{T}=\bar{s}_{4}-\bar{s}_{1} \\
& \operatorname{Max} \bar{Q}=\left(0.7 y_{121}+0.8 y_{122}+0.8 y_{231}\right. \\
& \left.+0.8 y_{232}+0.7 y_{341}+0.7 y_{342}\right) / 3 \\
& \text { S.T. } \\
& \qquad S^{\prime}:\left\{\begin{array}{l}
\bar{s}_{2}-\bar{s}_{1} \geq 4 y_{121}+2 y_{122} \\
\bar{s}_{3}-\bar{s}_{2} \geq 3 y_{231}+6 y_{232} \\
\bar{s}_{4}-\bar{s}_{3} \geq 5 y_{341}+7 y_{342} \\
y_{121}+y_{122}=1 \\
y_{231}+y_{232}=1 \\
y_{341}+y_{342}=1 \\
y_{121}+y_{122}, y_{231}, y_{232}, y_{341}, y_{342} \in\{0,1\}
\end{array}\right.
\end{aligned}
$$

By solving this model for three different objectives, the following results will be obtained: $\bar{C}^{*}=16, \bar{T}^{*}=10$, and $\underline{Q}^{*}=0.767$. In the last step, to obtain the final solution of the problem, the following goal programming model, Eq. (10), is formulated for this problem.

The optimal solution of model (15) is obtained as and, with an objective value of zero. Therefore, in this example, the target values are completely satisfied without any deviation. The associated time, cost and quality of this activity combination are $[6,10],[12,16]$, and $[0.767$, 0.867]. 


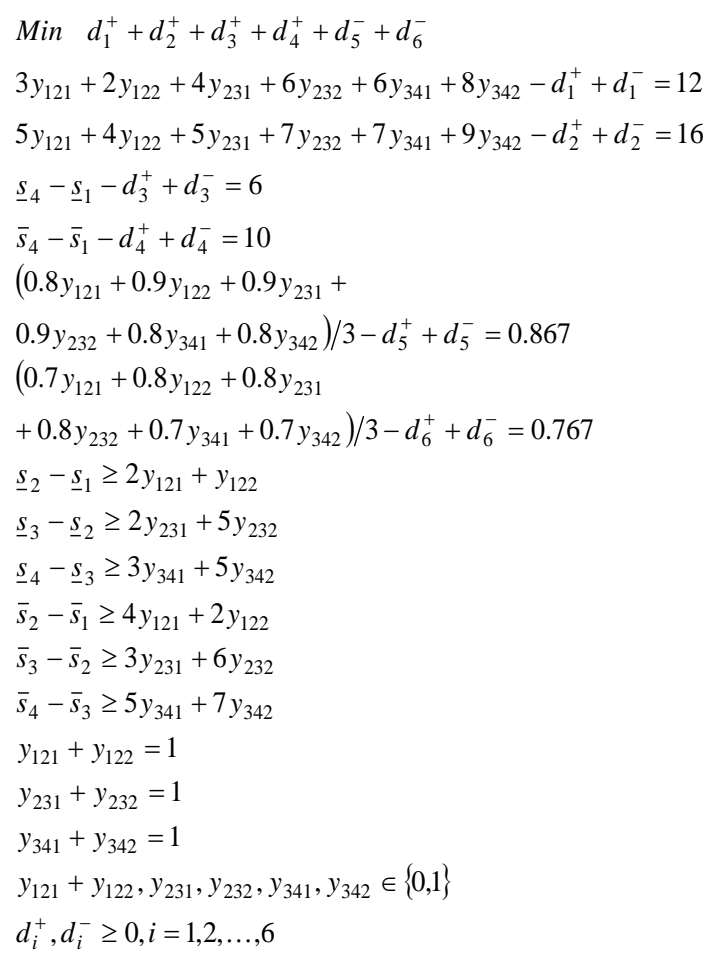

Example 2. Suppose that a project consists of eight activities based on data illustrated in table 2. The project's graph is illustrated in figure 2 .

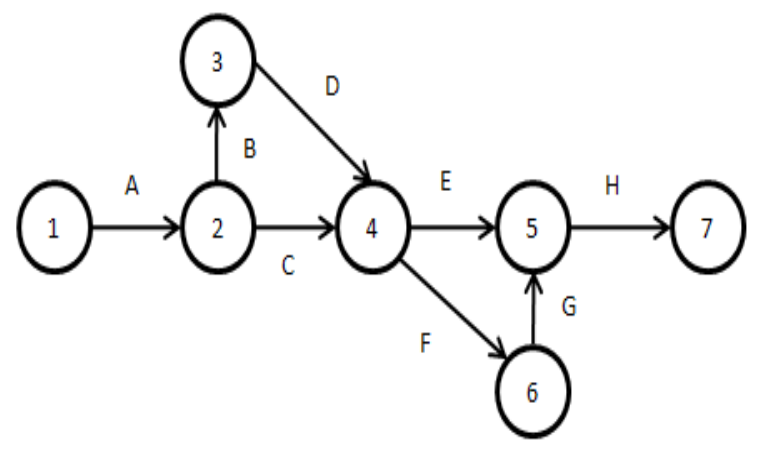

Figure 2. Project's graph

\begin{tabular}{|c|c|c|c|c|c|}
\hline \multirow[t]{2}{*}{ Activity Name } & \multirow[t]{2}{*}{ Predecessor } & \multicolumn{4}{|c|}{ Modes of execution } \\
\hline & & 1 & 2 & 3 & 4 \\
\hline A & - & $\left(\begin{array}{l}{[2,3]} \\
{[5,7]} \\
{[0.65,0.75]}\end{array}\right)$ & $\left(\begin{array}{l}{[3,4]} \\
{[4.5,6]} \\
{[0.7,0.8]}\end{array}\right)$ & $\left(\begin{array}{l}{[2.5,3.5]} \\
{[6,7.5]} \\
{[0.65,0.75]}\end{array}\right)$ & \\
\hline B & A & $\left(\begin{array}{l}{[1,1.5]} \\
{[3,4]} \\
{[0.6,0.7]}\end{array}\right)$ & $\left(\begin{array}{l}{[1.5,2.5]} \\
{[5,6]} \\
{[0.75,0.85]}\end{array}\right)$ & $\left(\begin{array}{l}{[1,2]} \\
{[4,5]} \\
{[0.7,0.8]}\end{array}\right)$ & \\
\hline $\mathrm{C}$ & A & $\left(\begin{array}{l}{[3,5]} \\
{[8,10]} \\
{[0.65,0.75]}\end{array}\right)$ & $\left(\begin{array}{l}{[2,4]} \\
{[10,12]} \\
{[0.75,0.85]}\end{array}\right)$ & $\left(\begin{array}{l}{[3,4.5]} \\
{[9,11]} \\
{[0.75,0.8]}\end{array}\right)$ & $\left(\begin{array}{l}{[2,3.5]} \\
{[9.11]} \\
{[0.7,0.8]}\end{array}\right)$ \\
\hline $\mathrm{D}$ & B & $\left(\begin{array}{l}{[1.5,3]} \\
{[4,6.5]} \\
{[0.7,0.8]}\end{array}\right)$ & $\left(\begin{array}{l}{[2,3]} \\
{[5,7]} \\
{[0.75,0.85]}\end{array}\right)$ & & \\
\hline $\mathrm{E}$ & $\mathrm{C}, \mathrm{D}$ & $\left(\begin{array}{l}{[4,6]} \\
{[8,11]} \\
{[0.7,0.75]}\end{array}\right)$ & $\left(\begin{array}{l}{[4,5.5]} \\
{[9,12]} \\
{[0.7,0.8]}\end{array}\right)$ & $\left(\begin{array}{l}{[3,4]} \\
{[10.13]} \\
{[0.75,0.8]}\end{array}\right)$ & \\
\hline $\mathrm{F}$ & C,D & $\left(\begin{array}{l}{[3,4]} \\
{[5,6.5]} \\
{[0.7,0.85]}\end{array}\right)$ & $\left(\begin{array}{l}{[2.5,4]} \\
{[6,8]} \\
{[0.7,0.8]}\end{array}\right)$ & $\left(\begin{array}{l}{[3,4]} \\
{[7,8.5]} \\
{[0.8,0.9]}\end{array}\right)$ & \\
\hline G & $\mathrm{F}$ & $\left(\begin{array}{l}{[1,2]} \\
{[3,6]} \\
{[0.6,0.7]}\end{array}\right)$ & $\left(\begin{array}{l}{[1.5,2]} \\
{[4,7]} \\
{[0.65,0.75]}\end{array}\right)$ & $\left(\begin{array}{l}{[2,3]} \\
{[3,5]} \\
{[0.7,0.8]}\end{array}\right)$ & \\
\hline $\mathrm{H}$ & $\mathrm{E}, \mathrm{G}$ & $\left(\begin{array}{l}{[6,8]} \\
{[15,20]} \\
{[0.75,0.85]}\end{array}\right)$ & $\left(\begin{array}{l}{[5,7]} \\
{[18,23]} \\
{[0.7,0.8]}\end{array}\right)$ & $\left(\begin{array}{l}{[5,7]} \\
{[20,25]} \\
{[0.8,0.9]}\end{array}\right)$ & \\
\hline
\end{tabular}

This example includes 5832 solutions which make it so hard to complete enumeration of its solutions to find the efficient solutions. Therefore, the proposed goal programming-based approach will be used. Optimizing three single objective functions $C, T$, and $Q$, the following
Table 2 results are obtained: $T^{*} \in[13,20.5], \quad C^{*} \in[50.5,69]$, and $Q^{*} \in[0.75,0.84375]$. Then the goal programming model is formulated as follows: 


$$
\begin{aligned}
& \sum_{i j \in E} \sum_{k \in M_{i j}}^{\operatorname{Mind}_{1}^{+}+d_{2}^{+}+d_{3}^{+}+d_{4}^{+}+d_{5}^{-}+d_{6}^{-}} y_{i j k}-d_{1}^{+}+d_{1}^{-}=50.5 \\
& \sum_{i j \in E} \sum_{k \in M_{i j}} \bar{c}_{i j k} y_{i j k}-d_{2}^{+}+d_{2}^{-}=69 \\
& \underline{\mathrm{s}}_{\mathrm{n}}-\underline{s}_{1}-d_{3}^{+}+d_{3}^{-}=13 \\
& \overline{\mathrm{s}}_{\mathrm{n}}-\overline{\mathrm{s}}_{1}-d_{4}^{+}+d_{4}^{-}=20.5 \\
& \sum_{i j \in E} \sum_{k \in M_{i j}} \underline{q}_{i j k} y_{i j k}-d_{5}^{+}+d_{5}^{-}=0.75 \\
& \sum_{i j \in E} \sum_{k \in M_{i j}} \bar{q}_{i j k} y_{i j k}-d_{6}^{+}+d_{6}^{-}=0.84375 \\
& \mathrm{~S} . \mathrm{T} . \\
& \mathrm{Y} \in \mathrm{FS} \\
& \mathrm{Y} \in \mathrm{FS}^{\prime} \\
& \mathrm{d}_{\mathrm{i}}^{+}, \mathrm{d}_{\mathrm{i}}^{-} \geq 0, i=1,2, \ldots, 6
\end{aligned}
$$

Where, FS and FS' are constructed as explained before. The solution of model (16) is as follows:

$$
\begin{aligned}
& y_{121}^{*}=y_{231}^{*}=y_{241}^{*}=y_{341}^{*}=y_{451}^{*}=y_{461}^{*}=y_{651}^{*}=y_{571}^{*}=1 \\
& T^{*}=[15,22], C^{*}=[51,71], Q^{*}=[0.75,0.84375]
\end{aligned}
$$

Now, suppose that the project manager takes different weights for time, cost and quality goals, as 5, 2, and 3 respectfully. Then the objective function of model (16) is replaced with the following one:

$$
\operatorname{Min} 5\left(d_{1}^{+}+d_{2}^{+}\right)+3\left(d_{3}^{+}+d_{4}^{+}\right)+2\left(d_{5}^{-}+d_{6}^{-}\right)
$$

Solving the objective function (17) with the same constraints as in model (16), the following results will be obtained:

$$
\begin{aligned}
& y_{121}^{*}=y_{231}^{*}=y_{244}^{*}=y_{341}^{*}=1 \\
& y_{451}^{*}=y_{461}^{*}=y_{651}^{*}=y_{571}^{*}=1, \\
& T^{*}=[14.5,19.5], C^{*}=[52,72], \\
& Q^{*}=[0.75,0.84375]
\end{aligned}
$$

In this phase, the project manager can make his/ her decision in regard to how different activities can be done.

\section{Conclusion}

Every task of an organization can be imagined as a project, which is a coordinated set of activities toward a common goal. Each project is a combination of a set of different activities which are connected to each other. Each project has several success criteria, among which the time, cost and quality of the project completion are very important. Project managers always try to find the best form of performing a project activity. In fact, each task can be done with different modes of time, cost and quality. Therefore, the time, cost and quality trade-off problems, which can have a major impact on the project success, are one of the most challenging factors in project management. On the other hand, the TCQT problems are always a subject of uncertainty. It is clear that determination of a task's exact time, cost and quality prior to its completion is a difficult task. In this paper, a model is proposed for TCQT problems, in which there are a set of different modes for each project, and the time, cost and quality of each mode are approximated in an interval. The proposed algorithm used a two-stage method to solve this problem. In stage one, the optimal solution of the problem is determined under the best and worst modes of activities. Then in the second stage, a goal programming model is developed to minimize the total deviation from the solutions of stage one. In each stage, it is sufficient to solve some linear integer problems, which are possible with ordinal applications, like Lingo. The proposed method provides a logical, feasible and solvable framework for the discrete TCQT problems under uncertainty. Future research can be focused on the different shapes of uncertainty in parameters approximation and development and comparison of different methods with the proposed one.

\section{References}

Abbasnia, R., Afshar, A., \& Eshtehardian, E. (2008). Time-Cost Trade-off Problem in Construction Project Management, Based on Fuzzy Logic. Journal of Applied Science 8(22), 4159-4165. http://dx.doi.org/10.3923/jas.2008.4159.4165

AbdelSalam, H. M., \& Bao, H. P. (2000). Solving the Project Time-Cost Trade-Off Problem through an Integrated Engineering-Computation Environment. Eleventh Annual Conference of the Production and Operations Management Society, POM, April 1-4, San Antonio, TX.

Abraham, A., Jain, L., \& Goldberg, R. (2006). Evolutionary Multiobjective Optimization: Theoretical Advances and Applications. New York: Springer-Verlag.

Babu, A. J. G., \& uresh, N. (1996).Project management with time, cost, and quality considerations, European Journal of Operational Research 88(2), 320-327. http://dx.doi.org/10.1016/0377-2217(94)00202-9

Blaszczyk, T., \& Nowak, M. (2009).The time-cost trade-off analysis in construction project using computer simulation and interactive procedure. Technological and Economic Development of Economy 15(4), 523-539. http://dx.doi.org/10.3846/1392-8619.2009.15.523-539

Charnes, A., \& Cooper, W.W. (1961). Management models and industrial applications of linear programming. New York: Wiley.

Cohen, I., Golany, B., \& Shtub, A. (2007). The Stochastic Time-Cost Trade-off Problem: A Robust Optimization Approach. NETWORKS 49(2), 175-188. http://dx.doi.org/10.1002/net.20153 
Demeulemeester, E., De Reyck, B., Foubert, B., Herroelen, W., \& Vanhoucke, M. (1998). New Computational Results On The Discrete Time/Cost Trade-off Problem In Project Networks. Journal of the Operational Research Society 49(11), 1153-1163. http://dx.doi.org/10.1057/palgrave.jors.2600634

Deng, J. L. (1982). Control problems of grey system. Systems and Control Letters 1(5), $288-294$. http://dx.doi.org/10.1016/S0167-6911(82)80025-X

Deng, J. L. (1989). Introduction to grey system theory. The Journal of Grey Theory 1, 1-24.

Iranmanesh, H., Skandari, M. R., \& Allahverdiloo, M. (2008). Finding Pareto Optimal Front for the Multi-Mode Time, Cost Quality Trade-off in Project Scheduling. Proceedings of World Academy of Science, Engineering and Technology 40, 346--350.

Jahanshahloo, G. R., HosseinzadehLotfi, F., Shoja, N., \& Tohidi, G. (2003).A method for solving 0-1 multiple objective linear programming problem using DEA. Journal of the Operations Research Society of Japan 46(2), 189-202.

Jahanshahloo, G. R., HosseinzadehLotfi, F., Shoja, N., \& Tohidi, G. (2005).A method for generating all efficient solutions of 0-1 multi-objective linear programming problem. Applied Mathematics and Computation 169(2), 874-886. http://dx.doi.org/10.1016/j.amc.2004.09.091

Kelly, J. (1961). Critical-path planning and scheduling: Mathematical basis. Operations Research 9(3), $296-320$. http://dx.doi.org/10.1287/opre.9.3.296

Khosrowshahi, F., Kaka, A. (2000).The Mathematics of Cost-Duration Trade-off Curves. Procurement Systems Symposium - Information and Communication in Construction Procurement, Santiago, Chile.

Kinson, R. (1999). Project management: cost, time and quality, two best guesses and a phenomenon, its time to accept other success criteria. International Journal of Project Management 17(6), 337-342. http://dx.doi.org/10.1016/S0263 $-7863(98) 00069-6$

Lewis, J. P. (2010). Project Planning, Scheduling, and Control: The Ultimate Hands-On Guide to Bringing Projects in on Time and on Budget. New York: McGraw-Hill.

Lin, Y., Chen, M. Y., \& Liu, S. (2004). Theory of grey systems: capturing uncertainties of grey information. Kybernetes 33(2), 196-218. http://dx.doi.org/10.1108/03684920410514139

Liu, F. H. F., Huang, C. C., \& Yen, Y. L. (2000). Using DEA to obtain efficient solutions for multi-objective-linear programs. European Journal of Operational Research 126(1), 51-68. http://dx.doi.org/10.1016/S03772217(99)00279-9

Liu, S., \& Lin, Y. (2006).Grey Information: Theory and Practical Applications (Advanced Information and Knowledge Processing). London, Springer.

Liu, S., \& Lin, Y. (2010).Grey Systems: Theory and Applications. Berlin, Heidelberg: Springer-Verlag. http://dx.doi.org/ 10.1007/978-3-642-13938-3

Lock, D. (2007). Project Management. Hampshire: Gower Publishing Company.

Mokhtari, H., \& Aghaie, A. (2009). The Effect of Price Discount on Time-Cost Trade-off Problem Using Genetic Algorithm. Engineering 1, 1-54. http://dx.doi.org/10.4236/eng.2009.11005

Nikoomaram, H., HosseinzadehLotfi, F. H., Jassbi, J., \& Shahriari, M. R. (2010). A New Mathematical Model for Time Cost Trade-off Problem with Budget Limitation Based on Time Value of Money. Applied Mathematical Sciences 4(63), 3107-3119.

Phillips, S. J. (1996). Project Management Duration/Resource Trade-off Analysis: an Application of the Cut Search Approach. Journal of the Operational Research Society 47(5), 697-701. http://dx.doi.org/10.1057/jors.1996.81

PMI: Project Management Institute, (2008). A Guide to the Project Management Body of Knowledge. Pennsylvania: Project Management Institute.

Rasmy, M. H., Abdelsalam, H. M., \& Ragab, R. (2008). Multi-objective Optimization of Time-Cost Trade-Off Analysis in Critical Chain Project Management Networks Using Pareto Simulated Annealing. 6th International Conference on Informatics and Systems (INFOS), 27-29 March, Cairo, Egypt.

Razavi Hajiagha, S. H., Amoozad Mahdiraji, H., \& Hashemi, S. S. (2014). A hybrid model of fuzzy goal programming and grey numbers in continuous project time, cost, and quality tradeoff. The International Journal of Advanced Manufacturing Technology 71(1-4), 117-126. http://dx.doi.org/10.1007/s00170-013-5463-2

Senouci, A., \& Rayes, Kh. (2009). Time-Profit Trade-Off Analysis for Construction Projects. Journal of Construction Engineering and Management 135(8), 718-725. http://dx.doi.org/10.1061/(ASCE)CO.1943-7862.0000031

Shahsavari Pour, N., Modarres, M., Tavakkoli-Moghaddam, R., \& Najafi, E. (2010).Optimizing a Multi-Objectives TimeCost-Quality Trade-Off problem by a New Hybrid Genetic Algorithm. World Applied Sciences Journal 10(3), $355-363$.

Vanhoucke, M. (2007).An electromagnetic time/cost trade-off optimization in project scheduling. Working paper, Faculty of Economics and Business Administration, Ghent University, Gent, Belgium, 28 April.

The article has been reviewed.

Received in June, 2013; accepted in February, 2015. 